\title{
Cytosolic flagellin receptor NLRC4 protects mice against mucosal and systemic challenges
}

\author{
FA Carvalho ${ }^{1,2}$, I Nalbantoglu ${ }^{3}$, JD Aitken ${ }^{1}$, R Uchiyama $^{1}$, Y Su$^{1}$, GH Doho $^{4}$, M Vijay-Kumar $^{1}$ \\ and AT Gewirtz ${ }^{1,4}$
}

Bacterial flagellin is a dominant innate immune activator of the intestine. Therefore, we examined the role of the intracellular flagellin receptor, NLRC4, in protecting the gut and/or driving inflammation. In accordance with NLRC4 acting through transcription-independent pathways, loss of NLRC4 did not reduce the rapid robust changes in intestinal gene expression induced by flagellin administration. Loss of NLRC4 did not alter basal intestinal homeostasis nor predispose mice to development of colitis upon administration of an anti-interleukin (IL)-10R monoclonal antibody. However, epithelial injury induced by dextran sulfate sodium in mice lacking NLRC4 resulted in a more severe disease, indicating a role for NLRC4 in protecting the gut. Moreover, loss of NLRC4 resulted in increased mortality in response to flagellate, but not aflagellate Salmonella infection. Thus, despite not being involved in rapid intestinal gene remodeling upon detection of flagellin, NLRC4-mediated inflammasome activation results in production of IL-1 $\beta$ and IL-18, two cytokines that protect mice from mucosal and systemic challenges.

\section{INTRODUCTION}

Bacterial flagellin can be recognized by two distinct signaling pathways of the innate immune system. ${ }^{1}$ Toll-like receptor (TLR)5-mediated detection of extracellular flagellin results in activation of canonical MyD88-mediated mitogen-activated protein kinase and nuclear factor- $\kappa \mathrm{B}$ signaling resulting in transcriptional activation of a panoply of genes related to inflammation and host defense. Detection of intracellular flagellin by NLRC4, also referred to as interleukin (IL)-converting enzyme protease-activating factor, results in activation of caspase- 1 resulting in post-translational processing/secretion of IL- $1 \beta$ and IL-18, ${ }^{2,3}$ TLR5 is functionally expressed on the basolateral surface of intestinal epithelial cells where it serves to activate pro-inflammatory gene expression upon translocation of bacteria beyond the epithelium. ${ }^{4}$ Although expression of TLR5 seems to be variable in hemopoietic cells, it is functionally present on intestinal CD11c + (likely dendritic cells or macrophages), thus being in accordance with the notion that flagellin is a dominant innate immune activator, capable of acting on multiple cell types, of the gut. ${ }^{5}$ In contrast, functionally significant expression of NLRC4 has yet to be observed in cells other than macrophages and was specifically not observed in cultured intestinal epithelial cells. ${ }^{6,7}$

Studies of TLR5-deficient mice (T5KO mice) have revealed that this receptor has a key role in protecting the gut from pathogens and, moreover, that loss of TLR5 renders mice unable to control their commensal microbiota, thus resulting in increased pro-inflammatory gene expression that can manifest as spontaneous colitis or eventuate in insulin resistance. ${ }^{8,9}$ In contrast, the extent to which NLRC4 has a role in protecting the intestine is largely unknown. Intestinal NLRC4 is functionally expressed in the intestine as mice deficient in NLRC4 (N4KO mice) lacked intestinal production of IL- $1 \beta$ and IL-18 in response to purified flagellin. ${ }^{7}$ Whether loss of NLRC4 alters the basal gut phenotype or the host response to a challenge, or both, remains undefined. Thus, the goal of this study was to define the role of NLRC4 in maintaining homeostasis of the gut and in protecting the host from challenges to this organ. We observed that NLRC4 was not required to maintain basal homeostasis, nor did its absence predispose mice to immune-mediated colitis. However, NLRC4 has a role in protecting the gut from chemical-induced acute colitis and mortality that results from dissemination of Salmonella beyond the gut.

\footnotetext{
${ }^{1}$ Center for Inflammation, Immunity and Infection, Department of Biology, Georgia State University, Atlanta, Georgia, USA. ${ }^{2}$ Department of Biology, Clermont Université, Université d'Auvergne, Inserm U1071, INRA USC, Clermont-Ferrand, France. ${ }^{3}$ Department of Pathology and Immunology, Washington University School of Medicine, St Louis, Missouri, USA. ${ }^{4}$ Department of Pathology and Winship Cancer Institute, Emory University School of Medicine, Atlanta, Georgia, USA.

Correspondence: AT Gewirtz (agewirtz@gsu.edu)

Received 9 August 2011; accepted 5 January 2012; published online 8 February 2012. doi:10.1038/mi.2012.8
} 


\section{RESULTS}

\section{Role of NLRC4 in mediating intestinal gene expression}

Bacterial flagellin is considered a dominant innate immune activator in the gut in that both intestinal epithelial cells and intestinal phagocytes are highly responsive to flagellin and unresponsive to the canonical TLR agonist LPS. ${ }^{6}$ Intestinal recognition of flagellin is mediated in part by TLR5, which is functionally expressed on the basolateral surface of intestinal epithelial cells $s^{4,10}$ and CD11c + lamina propria cells, presumed to be dendritic cells. ${ }^{5}$ In response to systemic challenge with purified flagellin or Salmonella, flagellin also activates the NLRC4 inflammasome, the expression of which has, so far, only been observed in macrophages. ${ }^{11,12}$ Mice lacking TLR5 (T5KO) are prone to developing spontaneous colitis, although whether colitis actually manifests in such mice is dependent on environmental factors, particularly the composition of the gut microbiota. ${ }^{9,13,14}$ However, regardless of whether T5KO mice exhibit spontaneous colitis, loss of TLR5 results in altered basal gene expression in the colon that can be most readily appreciated through use of microarray-based analysis of colon gene expression. ${ }^{13}$ To begin to investigate the role of NLRC4 in the gut, we bred mice engineered to lack NLRC4 (N4KO), generated on a C57BL/ 6 background, into our colony of T5KO mice thus allowing eventual generation of mice lacking NLRC4 and/or TLR5, as well as wild-type (WT) littermates that began life with common ancestry and, therefore, similar microbiotas. In contrast to our colony of $\mathrm{T} 5 \mathrm{KO}$ mice, $\mathrm{N} 4 \mathrm{KO}$ mice lacked indicators of spontaneous colitis such as loose stools, colomegaly, and splenomegaly, and, moreover, were indistinguishable from WT littermates based on myeloperoxidase (MPO) assay and histopathological analysis (Supplementary Figure 1a-c online). Moreover, microarray analysis performed in triplicate indicated that loss of NLRC4 had only minor effects on basal colonic gene expression (Supplementary Figure 1d online-full data set will be posted in GEO). Thus, in contrast to TLR5, loss of NLRC4-mediated recognition of flagellin may not have important consequences for intestinal phenotype in the absence of an overt challenge.

Next, to better understand the role of NLRC4 relative to TLR5 upon intestinal challenge, we first examined the roles of these receptors in regulating intestinal gene expression in vivo upon direct exposure to flagellin. Our approach was to administer flagellin in a manner known to be sufficient to activate both TLR5 and NLRC4, but to assay changes in gene expression at the mRNA level at a very early time $(1 \mathrm{~h})$ that was highly likely to reflect changes in gene transcription in cells that had detected flagellin (we reasoned that gene expression at later times would include many changes that resulted from responses to cytokines produced by directly responding cell). Mice (WT, T5KO, $\mathrm{N} 4 \mathrm{KO}$, and $\mathrm{T} 5 / \mathrm{N} 4-\mathrm{DKO}$ ), were systemically exposed to flagellin (10 $\mu$ g per mouse) through intraperitoneal injection, their colons harvested $1 \mathrm{~h}$ later, and mRNA immediately extracted. The $10 \mu \mathrm{g}$ dose was used because we previously demonstrated it was sufficient to robustly and specifically activate both NLRC4 and TLR5. Microarrays were performed on biological triplicates (i.e., three mice per condition-analyzed individually).
The complete set of results has been posted on National Library of Medicine's Gene Expression Omnibus (http://www. ncbi.nlm.nih.gov/geo/query/acc.cgi?acc=GSE34492), and a complete unbiased bioinformatics analysis can be found in Supplementary Data online. The main message of this data set, which can be most easily appreciated when data are viewed as heat map (Figure 1a) is that flagellin-induced changes in intestinal gene expression at this early time point are fully dependent on TLR 5 and largely independent of NLRC4. More specifically, none of the 226 genes that the significance analysis of microarrays algorithm indicated were induced by flagellin in WT mice, were significantly upregulated in mice lacking TLR5. Similar unresponsiveness to flagellin was observed in mice lacking both NLRC4 and TLR5, whereas, in contrast, mice lacking only NLRC4 exhibited robust flagellin-induced gene expression at this time point (195 genes significantly upregulated by significance analysis of microarrays algorithm), thus confirming that TLR5, rather than NLRC4, mediates this rapid induction of gene expression. Such TLR5-activated genes were analyzed using the KEGG (Kyoto Encyclopedia of Genes and Genomes) program to determine which pathways were significantly upregulated in response to flagellin (Table 1). Both WT and N4KO mice exhibited the same pattern of significant upregulated pathways. Selected genes from the microarray were examined by quantitative reverse transcription-PCR to confirm the microarray results (Figure $\mathbf{1 b}-\mathbf{e}$ ). Thus, the immediate gene expression remodeling that occurs in the intestine in response to detection of soluble flagellin is mediated by TLR 5 rather than by NLRC4.

\section{Role of NLRC4 in colitis}

Regardless of whether they are maintained in a manner that results in observable incidence of spontaneous colitis, loss of TLR5 predisposes mice to developing overt colitis upon ablation of IL-10 signaling ${ }^{9,13}$ and increases the severity of dextran sulfate sodium (DSS)-induced colitis. ${ }^{15}$ Thus, we investigated the extent to which loss of NLRC4 signaling might affect development of colitis under such conditions. First, we neutralized IL-10 signaling for 4 weeks through weekly injections of an IL$10 \mathrm{R}$ mAb. Such treatment was recently observed to uniformly result in robust colitis (100\% penetrance) in two distinctly generated strains of $\mathrm{T} 5 \mathrm{KO}$ mice. ${ }^{13}$ In contrast to $\mathrm{T} 5 \mathrm{KO}$ mice, and like WT mice, N4KO mice subjected to IL-10 blockade lacked evidence of colitis in that they continued to gain weight normally and showed neither gross nor histopathological evidence of colitis although, like WT mice, they showed increases in MPO activity in response to IL-10R neutralization (Figure 2a-e). Such IL-10R mAb-induced T5KO colitis was also associated with elevated levels of intestinal or systemic production of the inflammasome cytokines IL- $1 \beta$ or IL-18, respectively (Figure $2 \mathbf{f}$ and $\mathbf{g}$ ), which was not observed in $\mathrm{N} 4 \mathrm{KO}$ mice after IL-10R mAb treatment. A previous study has already shown that IL-10R mAb-induced colitis in T5KO mice correlates with colonic IL-1 $\beta$ production and requires the IL-1 receptor. ${ }^{16} \mathrm{We}$ hypothesized that such IL- $1 \beta$ production might reflect elevated expression/activity of NLRC4 that might result from loss of 
a

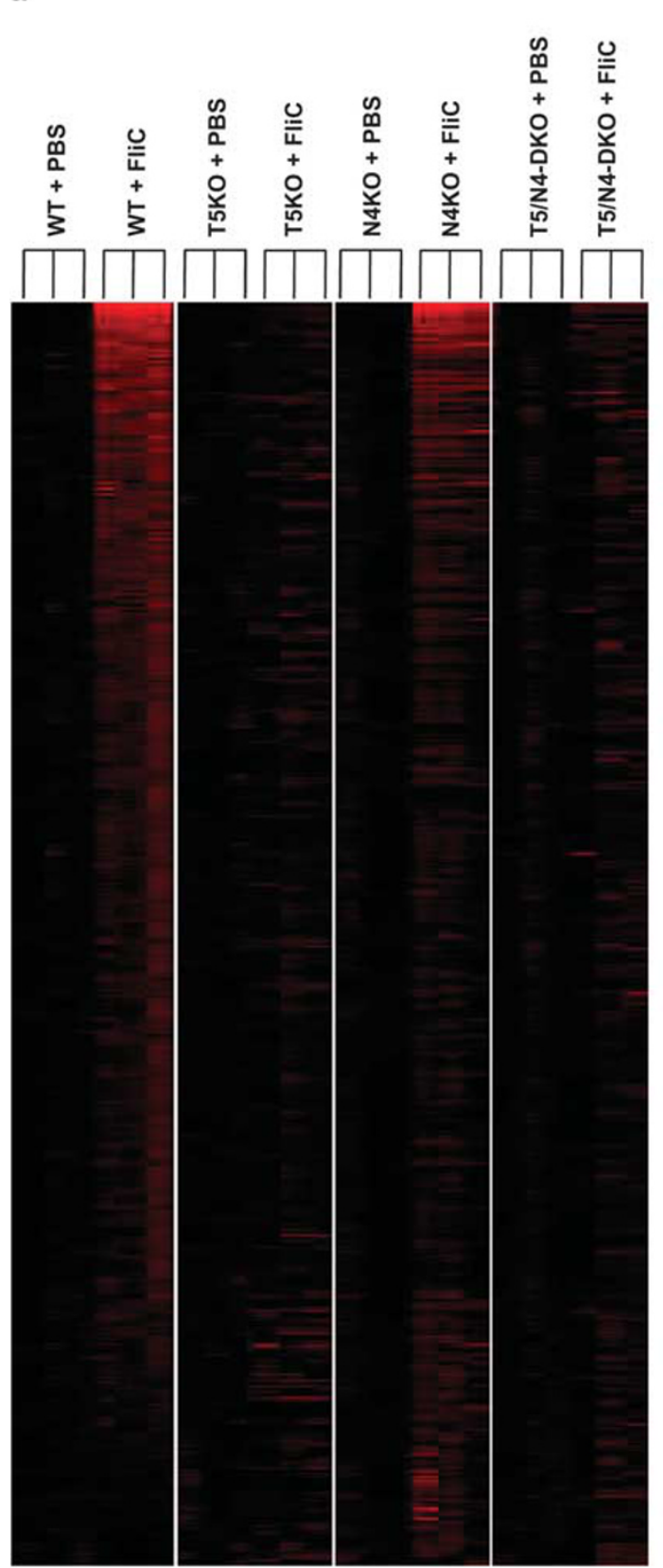

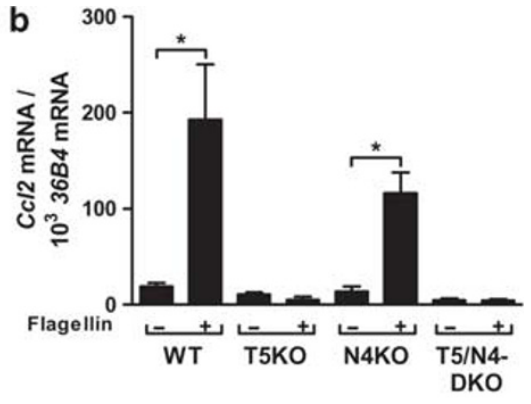
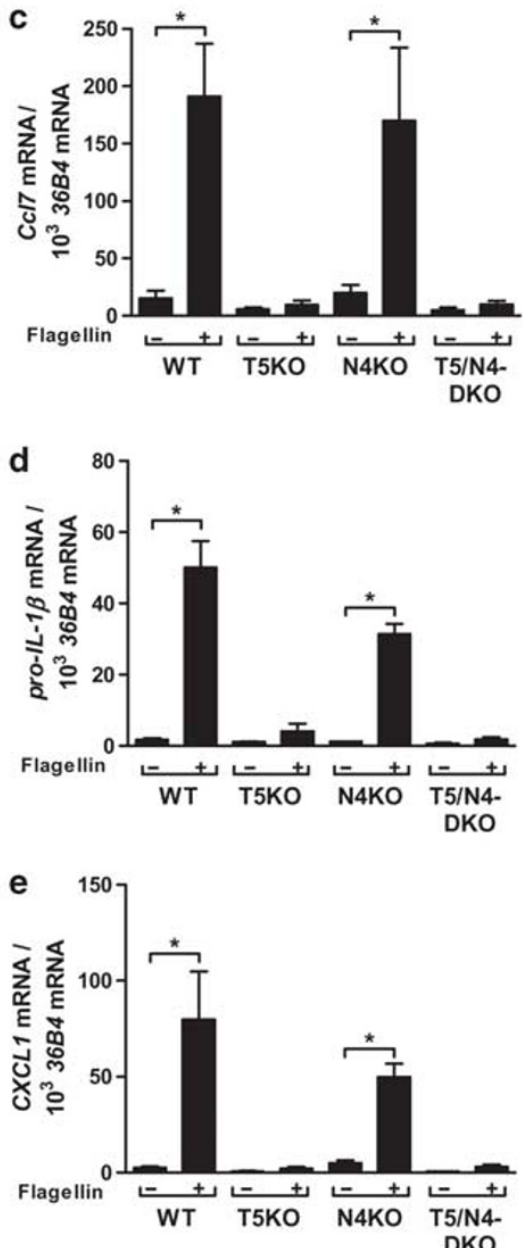

Figure 1 Flagellin-induced gene expression in colons of WT, TLR5-deficient (T5KO), NLRC4-deficient (N4KO), and TLR5/NLRC4-doubleKO (T5/N4-DKO) mice. Indicated strains of mice $(n=3)$ were administered $10 \mu \mathrm{g}$ of flagellin (FliC) in $0.2 \mathrm{ml}$ PBS through intraperitoneal injection. One-hour later, mice were killed, colons immediately removed, and mRNA extracted and subjected to microarray analysis of global gene expression. (a) Transcription profiles in the colons of WT, T5KO, N4KO, and T5/N4-DKO mice have been compared with the colons from vehicle-treated genotypematching mice. Heat map shows the results of all three mice per condition assayed. Illustration of genes induced with $>1.5$-fold change (in red) was built using the TreeView software (Berkeley, CA). (b-e) Quantitative reverse transcription-PCR (qRT-PCR) was used to confirm mRNA synthesis of selected genes. ${ }^{*} P<0.05$. PBS, phosphate-buffered saline; TLR5, Toll-like receptor-5; WT, wild type.

TLR5. However, although loss of NLRC4 upon a T5KO background modestly reduced the intestinal expression of IL-1 $\beta$ and IL-18 upon IL-10R mAb (Figure $2 \mathbf{f}$ and $\mathbf{g}$ ), mice lacking TLR5 and NLRC4 still developed robust colitis in response to IL-10R neutralization arguing against this possibility and suggesting that NLRP3 or another inflammasome pathway compensates for the lack of NLRC4.
We next examined the relative roles of TLR5 and NLRC4 in DSS-induced colitis, which results in focal destruction of the gut epithelium and subsequent colitis characterized by weight loss, bloody stools, splenomegaly, colomegaly, and increased colonic MPO levels. In accordance with the study by Ivison et al. ${ }^{15}$ T5KO mice exhibited more severe disease than did WT mice based on clinical-type indicators 
Table 1 Upregulated pathways in WT and N4KO mice shortly after flagellin injection

\begin{tabular}{lcc}
\hline Pathway & $\begin{array}{c}\text { WT (Fold } \\
\text { enrichment) }\end{array}$ & $\begin{array}{c}\text { N4KO (Fold } \\
\text { enrichment) }\end{array}$ \\
\hline $\begin{array}{l}\text { NOD-like receptor signaling } \\
\text { pathway }\end{array}$ & 16.7 & 18.0 \\
$\begin{array}{l}\text { Toll-like receptor signaling } \\
\text { pathway }\end{array}$ & 10.5 & 9.5 \\
$\begin{array}{l}\text { Cytosolic DNA-sensing pathway } \\
\begin{array}{l}\text { Cytokine-cytokine receptor } \\
\text { interaction }\end{array}\end{array}$ & 8.7 & 9.3 \\
$\begin{array}{l}\text { Chemokine signaling pathway } \\
\text { T-cell receptor signaling pathway }\end{array}$ & 7.8 & 5.6 \\
MAPK signaling pathway & 5.4 & 6.1 \\
\hline
\end{tabular}

MAPK, mitogen-activated protein kinase; PBS, phosphate-buffered saline; WT, wild type.

Gene expression level has been assessed by microarray using MouseWG-6 v2 chips (Affymetrix) in the colon of WT, T5KO, N4KO, and T5/N4-DKO mice $1 \mathrm{~h}$ after intraperitoneally flagellin injection in comparison with the matching genotype mice receiving only PBS. Upregulated genes, only observed in WT and N4KO mice, were then analyzed using the Database for Annotation, Visualization and Integrated Discovery (DAVID) tool (http://david.abcc.ncifcrf.gov/) for Kyoto Encyclopedia of Genes and Genomes (KEGG) pathways to determine which pathways were significantly upregulated after flagellin injection.

and histopathological analysis (Figure 3). N4KO mice were observed to be more even sensitive to this chemical colitogen exhibiting severe colitis in response to the relatively modest dose of 2\% DSS (Figure 3a-e). In particular, N4KO mice exhibited colonoscopic evidence of colitis and histopathological scoring confirmed greater severity of colitis in $\mathrm{N} 4 \mathrm{KO}$ mice (Figure 3f-g). The higher histological score of N4KO mice largely reflected that colitis in N4KO mice was transmural, whereas it was more focal in WT mice. Exposure of WT and $\mathrm{N} 4 \mathrm{KO}$ mice to a higher concentration of DSS (3.5\%) resulted in robust colitis in both WT and N4KO mice that did not significantly differ in its severity between these genotypes (data not shown). Exposure of WT, T5KO, N4KO, and T5/N4DKO mice to a lower concentration of DSS (1.25\%) did not result in discernible colitis within 7 days. However, prolonged DSS treatment eventuated in uniform death in mice lacking TLR5 and/or NLRC4, but not WT mice (Figure 3h), providing additional evidence that, like T5KO mice, N4KO mice exhibit more severe disease upon exposure to this chemical colitogen. To better understand how NLRC4 protected against DSS-induced disease, we measured cytokine production in this model (Figure 3i-k). Whereas loss of TLR5, by itself, resulted in reduced production of the neutrophil chemoattractant CXCL1 (perhaps explaining why histology/MPO showed reduced neutrophils but greater ulcerations), loss of NLRC4 resulted in reduced production of IL-18 suggesting that this inflammasome cytokine may protect against this chemical colitogen.

\section{Role of NLRC4 in Salmonella infection}

Intestinal recognition of flagellin protects against enteric and systemic disease in that infection by aflagellate Salmonella results in more severe intestinal and systemic disease in multiple mouse strains. ${ }^{16}$ Greater Salmonella-induced intestinal disease was also observed in mice lacking TLR5, although the extent to which the aflagellate phenotype can be phenocopied by deletion of TLR 5 varies considerably in different studies likely reflecting that the extent to which loss of TLR5 alters basal gene expression/phenotype is environment dependent. ${ }^{17}$ The above-described observation that loss of NLRC4 does not markedly affect basal intestinal gene expression, at least in our vivarium, minimizes this sort of caveat thus allowing us to use these KO mice to examine the role of NLRC4 in Salmonella infection. Mice (WT, T5KO, N4KO, and T5/N4-DKO) were subjected to the streptomycin/Salmonella model, which results in robust cecal inflammation characterized by edema and neutrophil recruitment within 2 days of infection. Whether assessed through cecum weight, MPO activity or histological scoring, loss of neither TLR5 nor NLRC4 did not have a significant effect on the robust inflammation in this model (Figure 4a-e). However, interestingly, loss of both NLRC4 and TLR5 or loss of MyD88, which will ablate signaling by TLR 5 and inflammasome cytokines (as well as other TLRs) dramatically reduced Salmonella-induced inflammation as assessed by MPO and histopathological scoring. Such protection against Salmonellainduced acute inflammation correlated with lack of production of the inflammasome cytokine IL-1 $\beta$, whereas increased CXCL1 was clearly not required for inflammation in this model (Figure $4 \mathbf{f}$ and $\mathbf{g}$ ). That ablation of the two known pathways of flagellin recognition phenocopies MyD88 deficiency in this model supports the notion that flagellin is a major pro-inflammatory actor during infection by flagellated pathogens and that inflammation can be driven by either innate immune detector capable of recognizing this protein.

Next, we compared WT and N4KO mice in response to low-dose oral challenge with Salmonella. As in the abovedescribed experiment, we used the SL3201 strain that has been used in many papers to study the role of flagella expression in Salmonella virulence. ${ }^{18,19}$ Use of a relatively low dose of $10^{6}$ colony-forming units (CFUs) resulted in death in $\sim 50 \%$ of WT mice and in $100 \%$ of N4KO mice (Figure 5a). Loss of NLRC4 on a TLR5-deficient background resulted in similarly increased lethality (100\%) within 3 weeks of infection (Figure 5b). Increasing the infectious dose by 100 -fold resulted in almost uniform lethality in both WT and N4KO mice, although the rate of death was significantly increased in N4KO mice (Figure 5c). To determine the extent to which the greater mortality exhibited by $\mathrm{N} 4 \mathrm{KO}$ mice might reflect increased Salmonella dissemination beyond the intestine, we examined bacterial loads in the spleen at days 3 and 7 . We found that bacterial loads in the spleen were indistinguishable on day 3 and significantly elevated on day 7 in N4KO mice, suggesting that loss of NLRC4 may result in more severe mortality through failure to control extraintestinal Salmonella replication (Figure 5d and e). Noting that loss of NLRC4 was not observed to significantly affect mortality in response to Salmonella strain SL1344, ${ }^{12}$ we infected our mice with this strain and also observed no difference between WT and N4KO 
a

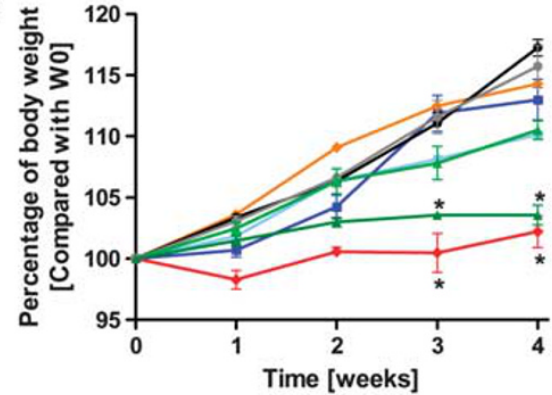

$\rightarrow$ WT + PBS

$\approx$ T5KO + PBS

$\rightarrow$ WT + IL-10R mAb

— T5KO + IL-10R mAb

$=\mathrm{N} 4 \mathrm{KO}+\mathrm{PBS} \quad \rightarrow \mathrm{N} 4 \mathrm{KO}+\mathrm{IL}-10 \mathrm{R}$ mAb

$\rightarrow$ T5/N4-DKO + PBS $\rightarrow$ T5/N4-DKO + IL-10R mAb b

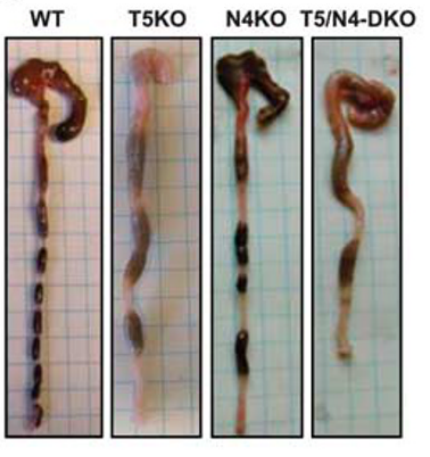

C

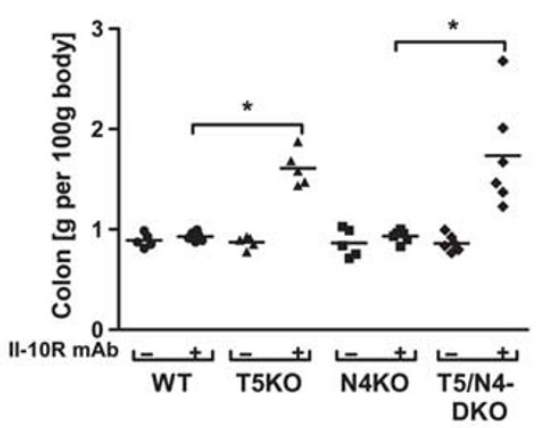

d

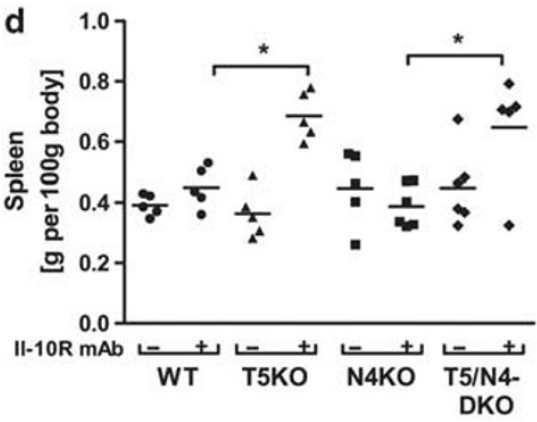

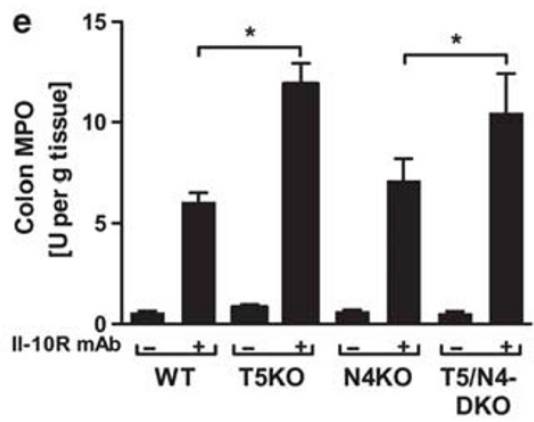

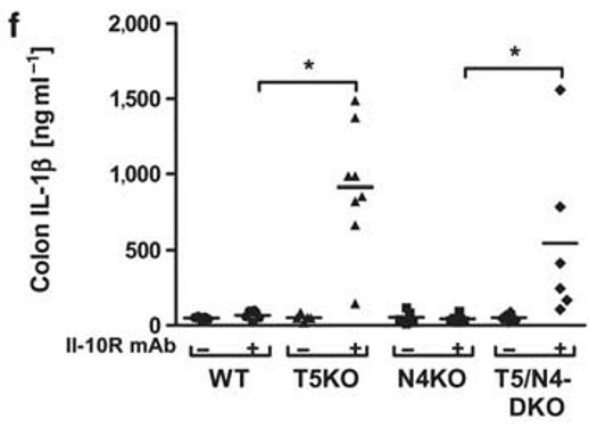

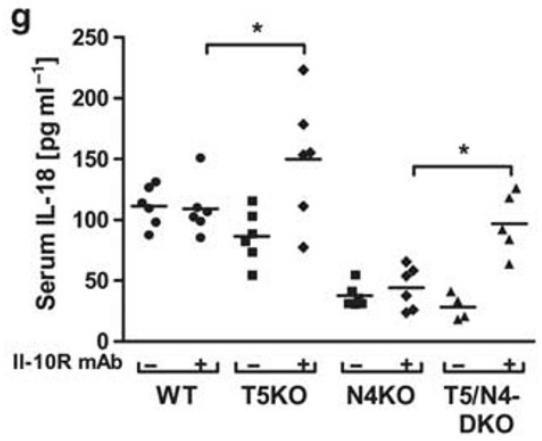

Figure 2 Induced intestinal inflammation in T5KO mice upon neutralization of the IL-10 pathway is NLRC4-independent. Wild-type, T5KO, N4KO, and T5/N4-DKO mice ( $n=5-6$ mice per group) were intraperitoneally injected weekly with $1 \mathrm{mg}$ IL-10R mAb or PBS (vehicle) for 4 weeks. (a) Body mass was monitored weekly during the treatment. (b) Gross picture of colon. (c) After the killing, the colon was isolated and mass measured. (d) Spleen mass. (e) Colonic MPO activity. (f) Colon was cultured for $24 \mathrm{~h}$, at which time supernatant was assayed for IL-1 $\beta$ by ELISA .The data are representative of two independent experiments. (g) Levels of IL-18 were measured in the sera by ELISA. Data show mean \pm s.e.m. of values obtained from individual mice. ${ }^{*} P<0.05$. ELISA, enzyme-linked immunosorbent assay; DKO, double knockout; IL, interleukin; MPO, myeloperoxidase; PBS, phosphate-buffered saline.

mice (data not shown), suggesting that the role of NLRC4 in response to this pathogen is dependent on bacterial strain. In addition to flagellin, NLRC4 is involved in detection of the Salmonella protein PrgJ. ${ }^{20}$ Thus, to determine the extent to which loss of NLRC4 reflected lost detection of flagellin, we infected WT and N4KO mice with an aflagellate mutant (SL3201 $\left.\mathrm{fliC}^{-} / \mathrm{flj}^{-}\right)$. No difference in mortality was observed between WT and N4KO mice (Figure 5f), indicating that the role of NLRC4 in protecting against Salmonella observed herein reflects recognition of flagellin during the course of the infection. Although the broadly appreciated role of IL-1 $\beta$ in host defense suggests that this cytokine likely contributes to
NLRC4-mediated defense against Salmonella infection, ${ }^{21,22}$ the role of IL-18 is less well studied. To begin to address whether the reduced ability of N4KO mice to withstand Salmonella infection reflected lost production of IL-18, we examined the extent to which loss of IL-18 signaling phenocopied that of N4KO mice. In particular, we examined Salmonella-induced mortality in mice engineered by Fantuzzi et al. ${ }^{23}$ to overexpress the natural antagonist of IL-18, IL-18-binding protein (IL-18 BP). These mice are maintained as heterozygous, thus producing IL-18 BP and WT control offspring and eliminating the potential of a non-specific difference resulting from paternally altered microbiota skewing our results. As shown 

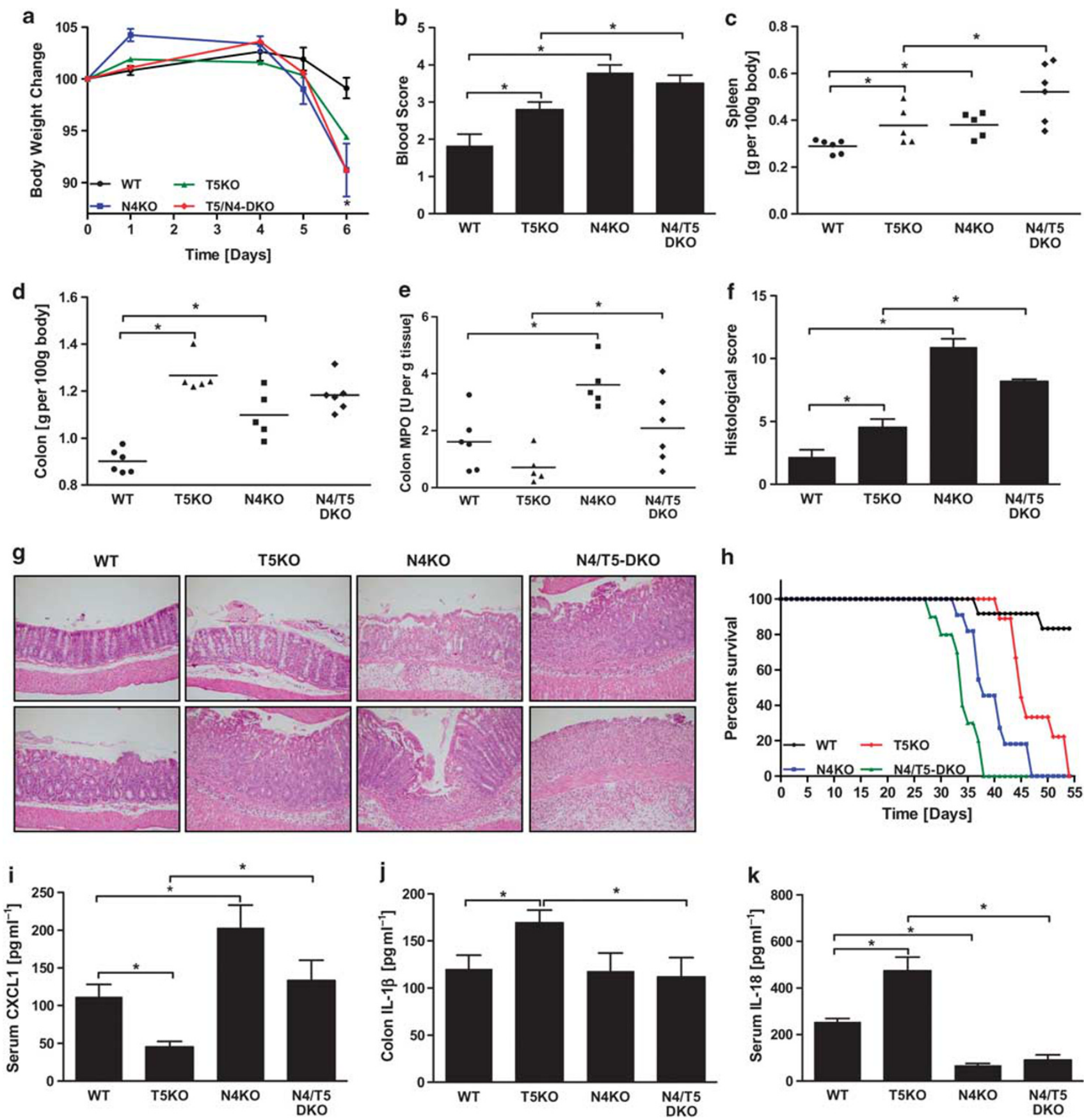

Figure 3 NLRC4-deficient mice exhibit more severe disease in DSS colitis model. Wild-type, T5KO, N4KO, and T5/N4-DKO mice ( $n=5-6$ mice per group) received water containing $2 \%$ DSS ad libitum throughout the experimental period (6 days). (a) Percentage of body weight change over the experimental period. (b) Rectal bleeding by assaying blood in stool. (c) After the killing, the spleen was isolated and mass measured. (d) Colon mass. (e) Colonic MPO activity. (f) Inflammation severity has been monitored in the colon by calculating a histological score as described in the "Methods" section. (g) Representative H\&E-stained histological observations of the mouse colon after 2\% DSS treatment. (h) DSS-induced mortality in mice that were administered a low dose of $1.25 \%$ DSS. (i) Levels of serum CXCL1 were measured by ELISA. (j) The colon was cultured for $24 \mathrm{~h}$, at which time supernatant was assayed for IL-1 $\beta$ by ELISA. The data are representative of three independent experiments. (k) Levels of IL-18 were measured in the sera by ELISA. Data show mean \pm S.e.m. of values obtained from individual mice. ${ }^{\star} P<0.05$. DSS, dextran sulfate sodium; ELISA, enzyme-linked immunosorbent assay; H\&E, hematoxylin and eosin; IL, interleukin; MPO, myeloperoxidase.

in Figure 5g, IL-18 BP mice exhibited increased severity to Salmonella infection reminiscent of that of N4KO mice suggesting that loss of IL-18 signaling mediates, in part, N4KO protection against Salmonella infection.

\section{DISCUSSION}

The notion that bacterial flagellin may be a major pro-inflammatory determinant in the gut suggested the possibility that blockade of innate immune recognition of flagellin might 
be a reasonable strategy to treat inappropriate intestinal inflammation. This concept is supported by the observation that, in some genetic backgrounds, carriage of a single-nucleotide polymorphism that results in 75\% loss of TLR5 function correlated with reduced incidence of Crohn's disease. ${ }^{24}$ However, complete loss of TLR5 in mice resulted in a tendency to develop spontaneous colitis. ${ }^{9}$ Although the incidence of such colitis was dependent on the composition of the microbiota in which the colony is maintained, T5KO mice were uniformly predisposed to developing colitis upon blockade of the anti-inflammatory cytokine IL-10 and developed more severe colitis on exposure to the chemical colitogen DSS. ${ }^{9,13,15}$ Finally, T5KO mice harboring a microbiota that did not result in colitis still exhibited elevated pro-inflammatory gene expression that may promote metabolic syndrome. ${ }^{8}$ Taken together, these observations in mice suggest that blockade of TLR5 in humans might result in unacceptable risks of disease development, including inducing the diseases that we along with others originally speculated it might prevent. Consequently, we have begun to explore the possibility that inhibition of the inflammasome pathway of flagellin recognition might have a therapeutic value in attenuating gut inflammation. Overall, our results do not broadly support the concept that specific inhibition of NLRC4 would be useful in treating/preventing inflammation. Rather, our observations shed light on the function of NLRC4 in the gut and serve as another example that blocking pro-inflammatory pathways can worsen outcomes of inflammatory diseases and increase the risk of severe infectious disease.

In contrast to the case for $\mathrm{T} 5 \mathrm{KO}$ mice, breeding of $\mathrm{N} 4 \mathrm{KO}$ mice into our colony resulted in neither evidence of spontaneous colitis nor markedly altered basal colonic gene expression. The lack of a significant role for NLRC4 in gut homeostasis seems unlikely to reflect an absolute inability of intestinal NLRC4 to recognize soluble flagellin as systemic exposure to flagellin induces NLRC4-dependent intestinal section of IL-1 $\beta$ and IL-18. ${ }^{7}$ However, it may simply reflect that NLRC4-mediated detection of soluble flagellin requires 100-1,000-fold higher concentrations of flagellin than does TLR5, and that the transient local breaches that occasionally occur in the gut epithelium are not sufficient to achieve concentrations of soluble flagellin sufficient to activate NLRC4. A related possibility would be that occasional breaches of the mucosa by bacteria themselves are not sufficient, or that perturbing bacteria do not persist long enough within intestinal phagocytes, to result in NLRC4 activation. In either case, our observation that TLR5 has a much greater role in basal gut gene expression than does NLRC4 is in accordance with the paradigm posited by Aderem and colleagues $^{1}$ that TLR5 deals with many challenges by flagellated bacteria, whereas NLRC4 activation serves as a "red alert" that a serious infection has occurred.

The association of inflammasome cytokines IL- $1 \beta$ and IL-18 with the extent of inflammation in clinical human gut inflammation and numerous murine colitis models, including T5KO mice, led us to speculate that deletion of NLRC4 might reduce inflammation in general and protect $\mathrm{T} 5 \mathrm{KO}$ mice from inflammation in particular. However, such protection was not observed. Rather, we observed increased severity of colitis in N4KO mice in response to DSS that eventuated in enhanced mortality. Such enhanced severity of colitis did not speak to the role of IL-1 $\beta$ in driving inflammation per se. Rather, that intestinal levels of IL- $1 \beta$ continued to correlate well with the severity of colitis, and T5/IL-1R-DKO mice are protected from colitis, suggests that ablation of NLRC4 simply eventuates in activation of the inflammasome through an alternate pathway that still results in production of IL- $1 \beta$. In contrast, multiple studies have observed that loss of the NLRP3 inflammasome actually reduced levels of IL- $1 \beta$ in DSS colitis, ${ }^{25,26}$ suggesting NLRC4 likely has a modulatory rather than dominant role in producing this cytokine. In contrast, the severe DSS-induced colitis in mice lacking NLRC4 occurred despite a dramatic absence of IL-18, suggesting that IL-18 may be protective in the gut and NLRC4 may have a non-redundant role in producing this inflammasome cytokine. Yet, the role of inflammasome cytokines in DSS colitis is apparently complex and environment dependent as NLRP3KO mice developed aggravated disease in one study and were protected in another. ${ }^{25,26}$ Thus, further development of pharmacological strategies to treat/prevent inflammation through inflammasome blockade require more basic understanding of the mechanisms that mediate inflammation/restitution in the gut.

That loss of NLRC 4 by itself did not reduce inflammatory pathology in any of the models studied parallels observations that the loss of TLR 5 by itself also provided no protection against inflammation in either IL-10 deficiency-induced colitis,${ }^{9,13}$ DSS colitis, ${ }^{15}$ or streptomycin/salmonella-induced intestinal inflammation. ${ }^{17}$ However, herein we observed that loss of both TLR 5 and NLRC4 dramatically reduced severity of inflammation in the latter model. Such lack of inflammation in T5/N4-DKO was reminiscent of the large reduction in inflammation seen in MyD88KO mice in this model. In contrast, MyD88KO mice exhibit markedly greater disease in response to DSS. ${ }^{27}$ The lack of an obvious role for TLR5/NLRC4 and MyD88 in protecting the gut in this model of acute inflammation may reflect that it lacks the severe epithelial damage

Figure 4 Flagellin is a major pro-inflammatory determinant of colitis induced by flagellated Salmonella. Wild-type, T5KO, N4KO, T5/N4-DKO, and MyD88KO mice ( $n=5-6$ mice per group) were pretreated with streptomycin (10 mg) and, $24 \mathrm{~h}$ later, infected orally with flagellate Salmonella typhimurium ( $10^{8} \mathrm{CFU}$ per mice). (a) After the killing (48h post infection), cecum was isolated and mass measured. (b) Cecum MPO activity. (c) Inflammation severity has been monitored in the cecum by calculating a histological score as described in the "Methods" section. (d) Gross picture of cecum. (e) Representative H\&E-stained histological observations of cecum after treatment. (f) Levels of serum CXCL1 were measured by ELISA. (g) Colon was cultured for $24 \mathrm{~h}$, at which time supernatant was assayed for IL-1 $\beta$ by ELISA. The data are representative of two independent experiments. ${ }^{\star} P<0.05$. CFU, colony-forming unit; DKO, double knockout; ELISA, enzyme-linked immunosorbent assay; H\&E, hematoxylin and eosin; MPO, myeloperoxidase. 
that characterizes the DSS model. In any case, considering that T5/N4-DKO mice should signal normally through other TLRs, the phenocopying of MyD88KO ${ }^{17,28}$ by T5/N4-DKO in this model is in accordance with the notion that flagellin is indeed a dominant pro-inflammatory determinant in the gut and that inability to signal in response to flagellin results

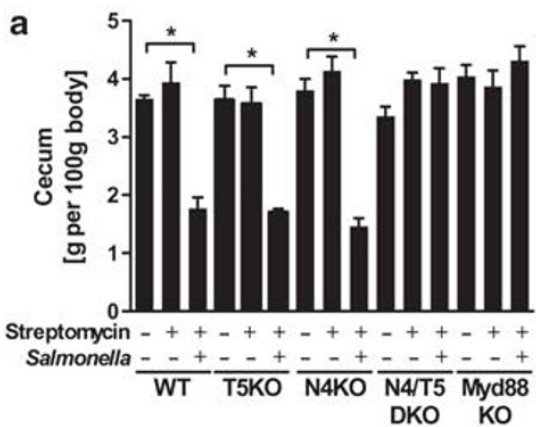

d
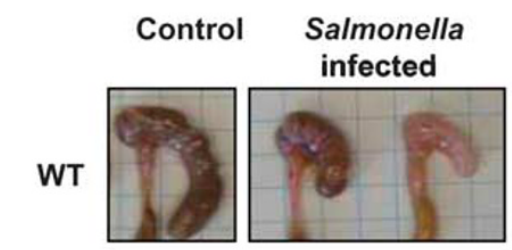

T5KO
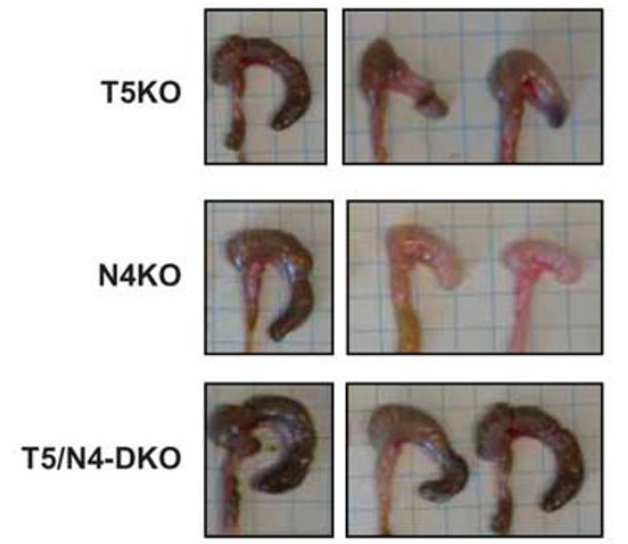

MyD88KO
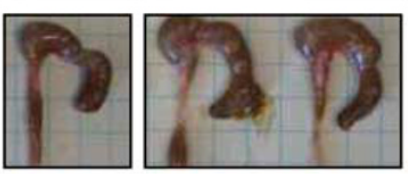

e
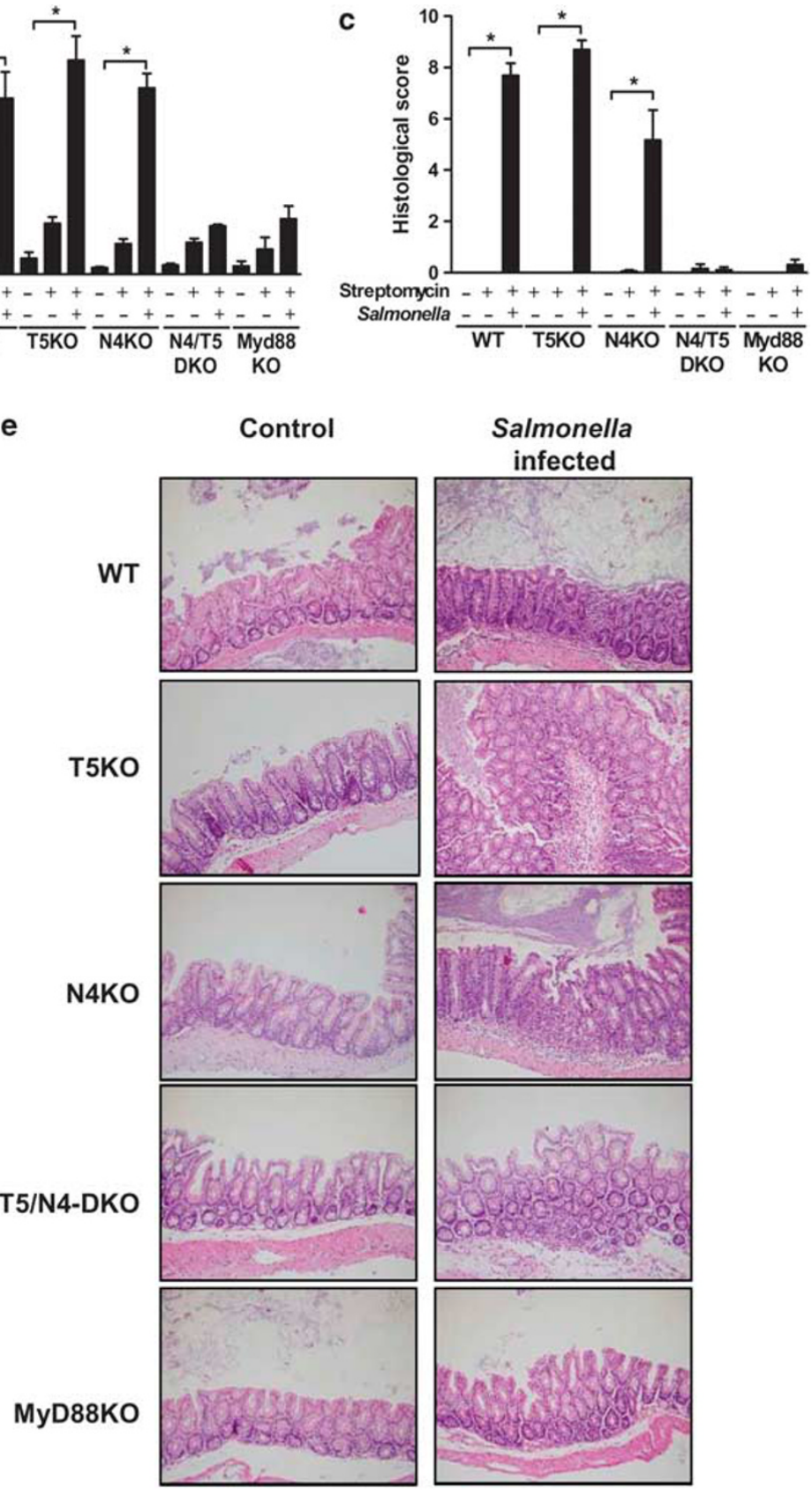
in failure to recruit immune cells in response to this pathogen. Although such "ignorance" of a pathogen seems likely to have dire consequences, it suggests the possibility that blocking all innate immune recognition of flagellin may have value in select scenarios.

The role of TLR5 in host defense against oral administration of Salmonella typhimurium, widely used as a model of typhoid-like illness, has been difficult to ascertain with T5KO mice as considerably different results have been reported by different investigators likely reflecting differences in basal phenotype and/or vivaria-specific differences in microbiota composition. ${ }^{14,17,29}$ We speculate that the lack of a basal phenotype in N4KO mice will prevent such variability. Herein, we observed that N4KO mice exhibited a clear increase in mortality in response to oral administration of Salmonella typhimurium. Such increased mortality was seen over a range of infectious doses, was specific for flagellated
Salmonella typhimurium, and correlated with greater splenic CFU at day 7, but not at day 3, post infection. One possible reason for why we saw increased splenic CFU at only the latter time is that there is delayed loss of gut defense/barrier integrity in $\mathrm{N} 4 \mathrm{KO}$ mice, perhaps reflecting a role for IL-18 in gut integrity, resulting in increased Salmonella translocation. However, given the reduced ability of $\mathrm{N} 4 \mathrm{KO}$ macrophages to kill Salmonella, perhaps a simpler explanation is that NLRC4 may be important in bacterial killing in the spleen but not the gut. In accordance, enhanced mortality of $\mathrm{N} 4 \mathrm{KO}$ mice was also observed in response to intraperitoneal infection (data not shown). Elegant pioneering studies by Miao et al. ${ }^{12} \mathrm{dem}-$ onstrated that a key means by which NLRC4 protects against systemic Salmonella infection is by driving macrophage pyroptosis that eventuates in bacterial killing by neutrophils. However, interestingly, Miao et al. only observed a protective role for NLRC4 against Salmonella engineered to maintain
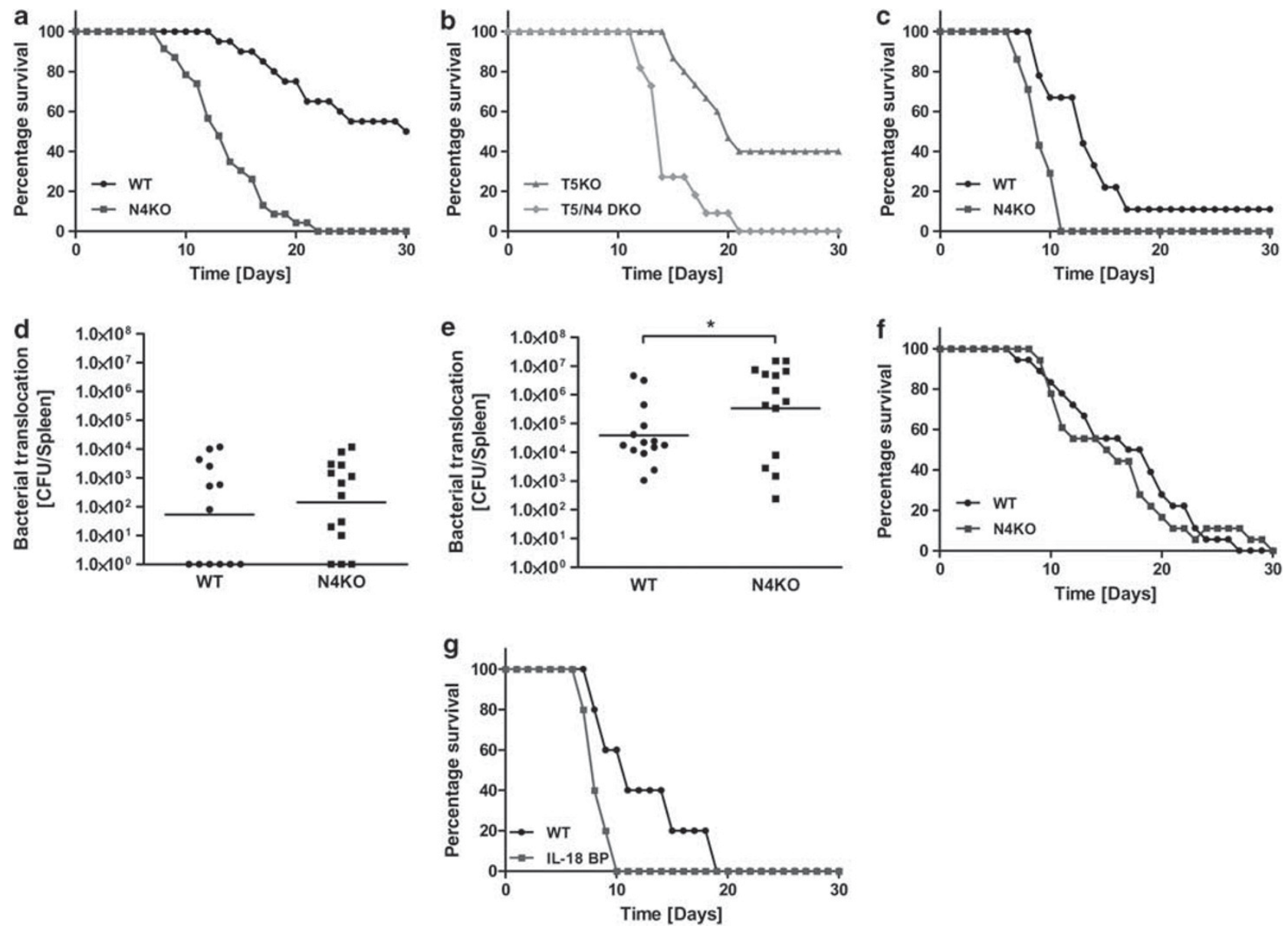

Figure 5 NLRC4-deficient mice are more susceptible to infection by flagellated Salmonella. Wild-type (in black), N4KO (in blue), T5KO (in green), and T5/N4-DKO (in red) mice ( $n=6-20$ mice per group) were orally infected with flagellate Salmonella typhimurium ( $10^{6}$ or $10^{8} \mathrm{CFU}$ per mice). (a) Salmonella-induced mortality using an infectious low-dose $\left(10^{6} \mathrm{CFU}\right.$ per mice) by gavage in WT and N4KO mice. (b) Salmonella-induced mortality using an infectious low-dose $\left(10^{6} \mathrm{CFU}\right.$ per mice) by gavage in T5KO and T5/N4-DKO mice. (c) Salmonella-induced mortality using an infectious dose of $10^{8} \mathrm{CFU}$ per mice by gavage in WT and N4KO mice. (d) Bacterial translocation by numbering Salmonella CFU present in the spleen at day 3 post infection. (e) Salmonella CFU present in the spleen at day 7 post infection. (f) WT and N4KO mice were infected by gavage with aflagellate Salmonella low-dose ( $10^{6} \mathrm{CFU}$ per mice) and monitored for 30 days. (g) Salmonella-induced mortality using an infectious dose of $10^{8} \mathrm{CFU}$ per mice by gavage in WT and IL-18BP mice (over-express the natural antagonist of IL-18, IL-18-binding protein). ${ }^{\star} P<0.05$. CFU, colony-forming unit; DKO, double knockout; IL, interleukin; WT, wild type. 
flagellin expression throughout infection. Given that we also did not see a difference between WT and N4KO mice when using their WT (SL1344) Salmonella strain, we speculate that the clear difference we see with our preferred WT (SL3201) strain may reflect reduced ability to rapidly turn off flagella expression in mice. Regardless, our observation indicates that any attempts to pharmacologically block NLRC4 signaling may increase the risk of severe infection.

\section{METHODS}

Mice. Generation of WT, T5KO, N4KO, and T5/N4-DKO was previously described. ${ }^{7}$ Mice overexpressing the natural antagonist of IL18, IL-18-binding protein (IL-18 BP), were engineered by Fantuzzi et al. ${ }^{23}$ (University of Colorado, Denver, CO) and generously given to us. Mice were bred and maintained at the Emory University and used between 6 and 8 weeks of age. All experiments involving animals were approved by the Emory and Georgia State Universities animal use committees.

Bacteria. WT Salmonella enterica serovar typhimurium (SL3201) and its isogenic mutant (aflagellate, phenotype: nonmotile; genotype: $\mathrm{fliC}^{-} / \mathrm{fljB}^{-}$) were grown in Luria-Bertani medium under microaerophilic conditions as described previously. ${ }^{16}$

Flagellin administration and mRNA preparation. Flagellin (FliC) from WT Salmonella enterica serovar typhimurium (SL3201, fljB ${ }^{-}$) was purified through sequential cation- and anion-exchange chromatography and purity was verified as described previously. ${ }^{4} \mathrm{WT}$, T5KO, N4KO, and T5/N4-DKO mice $(n=6)$ were given either $0.2 \mathrm{ml}$ phosphate-buffered saline (PBS) or flagellin $(10 \mu \mathrm{g}$ per mouse in $0.2 \mathrm{ml} \mathrm{PBS})$ intraperitoneally. After $1 \mathrm{~h}$, mice were killed and colons taken and stored in RNAlater (Invitrogen, Carlsbad, CA) for 1 day. Total mRNA was isolated from colonic tissues using TRIzol (Invitrogen) and purified using the RNeasy Plus Mini kit (Qiagen, Valencia, CA) according to the manufacturer's instructions. ${ }^{6}$

Microarray. Microarray analyses were performed at the Emory Biomarker Microarray Core. In brief, mRNA samples were reversetranscribed, amplified, labeled, and used to probe MouseWG-6 v2 chips purchased from Affymetrix (Santa Clara, CA). Samples were assayed using a Molecular Devices Gene Pix (4100A) (Molecular Devices, Silicon Valley, CA) and raw fluorescence readings were processed by an algorithm designed to reduce spurious readouts of gene activation. Microarray data were quantile normalized using freely available scripts written in R (http://R-project.org). Significantly altered genes were identified using Significance Analysis of Microarrays algorithm and assessed by hierarchal clustering and principle component analysis using Spotfire Decision Site for Functional Genomics software (Tibco, Somerville, MA) to determine relatedness of gene expression patterns resulting from loss of NLRC4. Upregulated genes, only observed in WT and N4KO mice, were then analyzed using the Database for Annotation, Visualization and Integrated Discovery (DAVID) tool (http://david.abcc.ncifcrf.gov/) for KEGG pathways to determine which pathways were significantly upregulated after flagellin injection.

Quantitative reverse transcription-PCR. RNA was quantified as described previously. ${ }^{13}$ In brief, previous purified total mRNA were measured in Realplex ${ }^{4}$ (Eppendorf, Hauppauge, NY) using QuantiFast SYBRGreen reverse transcription-PCR Kit (Qiagen) with specific mouse oligonucleotides. The sense and antisense oligonucleotides used were $36 B 4$, 5' -TCCAGGCTTTGGGCATCA-3' and 5' -CTTTATCAGCTGCACA TCACTCAGA-3'; pro- $I L-1 \beta, 5^{\prime}$-TTGACGGACCCCAAAAGATG- ${ }^{\prime}$ and 5' -AGAAGGTGCTCATGTCCTCAT-3'; CXCL1, 5' -TCGCGAG GCTTGCCTTGACC- ${ }^{\prime}$ and $5^{\prime}$-AGACGGTGCCATCAGAGCAG- ${ }^{\prime}$; $c c l 2,5$,-GATCACCAGCAGCAGGTGTCC-3' and 5' -CAAAGGTG
CTGAAGACCTTAGG-3'; $c c l 7$, 5' - AGAAACAAAAGATCCCCA AGAGG-3' and 5' - GGGGTTTTCATGTCTAAGTATGC-3', respectively. All results were normalized to the unaffected housekeeping $36 B 4$ gene.

Administration of anti-IL-10R monoclonal antibody (IL-10R mAb). IL-10R-neutralizing $\mathrm{mAb}$ (1B1.3a) was purchased from BioXcell (West Lebanon, NH). WT, N4KO, and T5/N4-DKO mice $(n=6)$ were treated with $1 \mathrm{mg}$ of IL-10R mAb (intraperitoneally) weekly for 4 weeks as described previously. ${ }^{13,30}$ As a control, mice that did not receive IL-10R $\mathrm{mAb}$ were injected with sterile PBS. Mouse body mass was measured weekly. After the killing, colitis severity was assessed by measuring spleen and colon weight, as well as colonic MPO activity and cytokine production.

DSS treatment. WT and N4KO male mice, aged 6-8 weeks ( $\approx 22 \mathrm{~g})$, received $2 \%(\mathrm{w} / \mathrm{v})$ DSS (molecular weight $=36,000-50,000 \mathrm{kDa}, \mathrm{MP}$ Biomedicals, Solon, $\mathrm{OH}$ ) in drinking water for 6 days to induce colon injury. Rectal bleeding was assessed by Hemoccult II test (SKD SARL). Blood score ranges from 0 (no blood) to 4 (visible rectal bleeding). Colitis progression was also assessed by colonoscopy to look for ulcerations.

Streptomycin pretreated Salmonella induced gastroenteritis. WT Salmonella enterica serovar typhimurium (SL3201) were grown and streptomycin-pretreated Salmonella induced gastroenteritis was induced in WT, N4KO, T5/N4-DKO, and MyD88KO mice as described previously. ${ }^{31}$ In brief, mice were fasted for $4 \mathrm{~h}$ before streptomycin treatment (10 mg per mice by gavage). One day after, mice were infected by gavage using $10^{8} \mathrm{CFU}$ of Salmonella per mice. Mice were then killed $48 \mathrm{~h}$ post infection and cecal inflammation was assessed.

Colon culture. After the killing, colons $(1 \mathrm{~cm})$ were removed, cut open longitudinally, washed in Hank's balanced salt solution and cultured in RPMI 1640 medium containing 1\% penicillin and streptomycin. ${ }^{32}$ After $24 \mathrm{~h}$ incubation at $37^{\circ} \mathrm{C}$ with $5 \% \mathrm{CO}_{2}$, the supernatants were centrifuged at $4^{\circ} \mathrm{C}$ and used for assaying cytokines by enzyme-linked immunosorbent assay.

Tissue MPO assay. Neutrophil influx in tissue was accessed by assaying the enzymatic activity of MPO, a widely used marker for neutrophils. In brief, tissue $\left(50 \mathrm{mg} \mathrm{ml}^{-1}\right)$ was thoroughly washed in PBS and homogenized in $0.5 \%$ hexadecyltrimethylammonium bromide (Sigma, St Louis, MO) in $50 \mathrm{mM}$ PBS ( $\mathrm{pH} 6.0$ ), freeze-thawed three times, sonicated, and centrifuged. MPO was assayed in the clear supernatant by adding $1 \mathrm{mg} \mathrm{ml}^{-1}$ dianisidine dihydrochloride (Sigma) and $5 \times 10^{-4} \% \mathrm{H}_{2} \mathrm{O}_{2}$ and the change in optical density measured at $450 \mathrm{~nm}$. Human neutrophil MPO (Sigma) was used as standard. One unit of MPO activity was defined as the amount that degraded $1.0 \mu \mathrm{mol}$ of peroxide per min at $25^{\circ} \mathrm{C} .^{33}$

Enzyme-linked immunosorbent assay. IL-1 $\beta$ and CXCL1 enzymelinked immunosorbent assays were performed using kits purchased from R\&D Systems (Minneapolis, MN) according to the manufacturer' instructions. The minimum detectable doses $\left(\mathrm{pg} \mathrm{ml}^{-1}\right)$ were CXCL1 (15.6), and IL-1 $\beta$ (15.6). IL-18 was quantitated with R\&D Systems quantikine kit (minimum detection was $25 \mathrm{pg} \mathrm{ml}^{-1}$ ).

Histology. After the killing, cecum or colon was fixed for $24 \mathrm{~h}$ in $10 \%$ buffered formalin at room temperature and then subjected to hematoxylin and eosin staining on tissue sections of $5 \mu \mathrm{m}$ thickness. Hematoxylin and eosin-stained slides were scored by a pathologist (IN) blinded to the study protocol as described previously. ${ }^{34}$ In brief, slides were scored for the presence/absence of active inflammation, the intensity of inflammation (average number of neutrophils and the number of fields that were involved), the extent of inflammation (mucosa, submucosa, or serosa), the presence/absence of ulceration, architectural disarray, and the pattern of involvement. 
Low-dose oral Salmonella infection. WT Salmonella enterica serovar typhimurium (SL3201) and its aflagellate isogenic mutant, $\mathrm{fliC}^{-} / \mathrm{flj}^{-}$, were used to infect WT and N4KO mice. In brief, mice were fasted $4 \mathrm{~h}$ and infected orally by gavage with either flagellate or aflagellate Salmonella typhimurium $\left(1-10^{6}\right.$ or $1-10^{8} \mathrm{CFU}$ per mouse). Mice were killed after loss of $>30 \%$ of their initial body weight. Salmonella typhimurium translocation was also measured at days 3 and 7 post infection by counting the Salmonella CFU present in the spleen after plating on Luria-Bertani agar plate.

Statistical analysis. Significance was determined using Student's $t$-test, the Mann-Whitney $t$-test or one-way analysis of variance (GraphPad Prism software; GraphPad, La Jolla, CA). Differences were noted as significant ${ }^{\star} P<0.05$.

SUPPLEMENTARY MATERIAL is linked to the online version of the paper at http://www.nature.com/mi

\section{ACKNOWLEDGMENTS}

This work was supported by NIH Grants DK061417 and DK083890 to AT Gewirtz. FA Carvalho and M Vijay-Kumar are recipients of Research Fellowship and Career Development awards from the Crohn's and Colitis Foundation of America, respectively. We also acknowledge NIH Digestive Disease Research and Development Center (DDRDC) Grants to Emory University (DK064399). We thank Daniel Moore, Catherine Paul, and Sindhu Srinivasan for excellent technical support.

\section{DISCLOSURE}

The authors declared no conflict of interest.

(C) 2012 Society for Mucosal Immunology

\section{REFERENCES}

1. Miao, E.A., Andersen-Nissen, E., Warren, S.E. \& Aderem, A. TLR5 and Ipaf: dual sensors of bacterial flagellin in the innate immune system. Semin. Immunopathol. 29, 275-288 (2007).

2. Franchi, L. et al. Cytosolic flagellin requires Ipaf for activation of caspase-1 and interleukin 1 beta in salmonella-infected macrophages. Nat. Immunol. 7, 576-582 (2006)

3. Miao, E.A. et al. Cytoplasmic flagellin activates caspase- 1 and secretion of interleukin 1 beta via Ipaf. Nat. Immunol. 7, 569-575 (2006).

4. Gewirtz, A.T., Navas, T.A., Lyons, S., Godowski, P.J. \& Madara, J.L. Cutting edge: bacterial flagellin activates basolaterally expressed TLR5 to induce epithelial proinflammatory gene expression. J. Immunol. 167, 1882-1885 (2001)

5. Uematsu, S. et al. Detection of pathogenic intestinal bacteria by Toll-like receptor 5 on intestinal CD11 c+ lamina propria cells. Nat. Immunol. 7, 868-874 (2006).

6. Carvalho, F.A., Aitken, J.D., Gewirtz, A.T. \& Vijay-Kumar, M. TLR5 activation induces secretory interleukin-1 receptor antagonist (sIL-1Ra) and reduces inflammasome-associated tissue damage. Mucosal Immunol. 4, 102-111 (2011).

7. Vijay-Kumar, M., Carvalho, F.A., Aitken, J.D., Fifadara, N.H. \& Gewirtz, A.T. TLR5 or NLRC4 is necessary and sufficient for promotion of humoral immunity by flagellin. Eur. J. Immunol. 40, 3528-3534 (2010).

8. Vijay-Kumar, M. et al. Metabolic syndrome and altered gut microbiota in mice lacking Toll-like receptor 5. Science 328, 228-231 (2010).

9. Vijay-Kumar, M. et al. Deletion of TLR5 results in spontaneous colitis in mice. J. Clin. Invest. 117, 3909-3921 (2007).

10. Rhee, S.H. et al. Pathophysiological role of Toll-like receptor 5 engagement by bacterial flagellin in colonic inflammation. Proc. Natl Acad Sci. USA 102, 13610-13615 (2005).

11. Franchi, L., Eigenbrod, T., Munoz-Planillo, R. \& Nunez, G. The inflammasome: a caspase-1-activation platform that regulates immune responses and disease pathogenesis. Nat. Immunol. 10, 241-247 (2009).

12. Miao, E.A. et al. Caspase-1-induced pyroptosis is an innate immune effector mechanism against intracellular bacteria. Nat. Immunol. 11, 1136-1142 (2010).

13. Carvalho, F.A. et al. Interleukin-1 \{beta\} (IL-1\\{beta \\}) promotes susceptibility of Toll-like receptor 5 (TLR5) deficient mice to colitis. Gut (2012) (in press).

14. Letran, S.E. et al. TLR5-deficient mice lack basal inflammatory and metabolic defects but exhibit impaired CD4 T cell responses to a flagellated pathogen. J. Immunol. 186, 5406-5412 (2011).

15. Ivison, S.M. et al. TLR5 is not required for flagellin-mediated exacerbation of DSS colitis. Inflamm. Bowel Dis. 16, 401-409 (2010).

16. Vijay-Kumar, M. et al. Flagellin suppresses epithelial apoptosis and limits disease during enteric infection. Am. J. Pathol. 169, 1686-1700 (2006).

17. Vijay-Kumar, M. et al. Toll-like receptor 5-deficient mice have dysregulated intestinal gene expression and nonspecific resistance to Salmonella-induced typhoid-like disease. Infect Immunol. 76, 1276-1281 (2008).

18. Carsiotis, M., Weinstein, D.L., Karch, H., Holder, I.A. \& O'Brien, A.D. Flagella of Salmonella typhimurium are a virulence factor in infected C57BL/6J mice. Infect Immunol. 46, 814-818 (1984).

19. Schmitt, C.K., Darnell, S.C., Tesh, V.L., Stocker, B.A. \& O'Brien, A.D. Mutation of flgM attenuates virulence of Salmonella typhimurium, and mutation of fliA represses the attenuated phenotype. J. Bacteriol. 176, 368-377 (1994).

20. Miao, E.A. et al. Innate immune detection of the type III secretion apparatus through the NLRC4 inflammasome. Proc. Natl Acad. Sci. USA 107, 3076-3080 (2010).

21. Broz, P. \& Monack, D.M. Molecular mechanisms of inflammasome activation during microbial infections. Immunol. Rev. 243, 174-190 (2011).

22. Miao, E.A. \& Rajan, J.V. Salmonella and caspase-1: a complex interplay of detection and evasion. Front Microbiol. 2, 85 (2011).

23. Fantuzzi, G. et al. Generation and characterization of mice transgenic for human IL-18-binding protein isoform a. J. Leukoc. Biol. 74, 889-896 (2003).

24. Gewirtz, A.T. et al. Dominant-negative TLR5 polymorphism reduces adaptive immune response to flagellin and negatively associates with Crohn's disease. Am. J. Physiol. Gastrointest. Liver Physiol. 290, G1157-1163 (2006).

25. Bauer, C. et al. Colitis induced in mice with dextran sulfate sodium (DSS) is mediated by the NLRP3 inflammasome. Gut 59, 1192-1199 (2010).

26. Zaki, M.H. et al. The NLRP3 inflammasome protects against loss of epithelial integrity and mortality during experimental colitis. Immunity $\mathbf{3 2}$, 379-391 (2010).

27. Rakoff-Nahoum, S., Paglino, J., Eslami-Varzaneh, F., Edberg, S. \& Medzhitov, R. Recognition of commensal microflora by toll-like receptors is required for intestinal homeostasis. Cell 118, 229-241 (2004).

28. Keestra, A.M. et al. Early, MyD88-dependent induction of interleukin-17A expression during Salmonella colitis. Infect Immunol. 79, 3131-3140 (2011).

29. Hayashi, F. et al. The innate immune response to bacterial flagellin is mediated by Toll-like receptor 5. Nature 410, 1099-1103 (2001).

30. Kullberg, M.C. et al. IL-23 plays a key role in Helicobacter hepaticusinduced T cell-dependent colitis. J. Exp. Med. 203, 2485-2494 (2006).

31. Barthel, M. et al. Pretreatment of mice with streptomycin provides a Salmonella enterica serovar Typhimurium colitis model that allows analysis of both pathogen and host. Infect Immunol. 71, 2839-2858 (2003).

32. Vijay-Kumar, M. et al. Activation of toll-like receptor 3 protects against DSS-induced acute colitis. Inflamm. Bowel Dis. 13, 856-864 (2007).

33. Castaneda, F.E. et al. Targeted deletion of metalloproteinase 9 attenuates experimental colitis in mice: central role of epithelial-derived MMP. Gastroenterology 129, 1991-2008 (2005)

34. Onyeagocha, C. et al. Latent cytomegalovirus infection exacerbates experimental colitis. Am. J. Pathol. 175, 2034-2042 (2009). 